\title{
Antineutrophil cytoplasm antibody in crescentic glomerulonephritis
}

\author{
M D S WALTERS, C O S SAVAGE, ${ }^{*}$ M J DILlON, C M LOCKWOOD,$*$ \\ AND T M BARRATT
}

Department of Paediatric Nephrology, Institute of Child Health, London, and *Department of Medicine, Royal Postgraduate Medical School, Hammersmith Hospital, London

SUMMARY Antineutrophil cytoplasm antibody (ANCA) has been reported in the sera of adults with Wegener's granulomatosis and microscopic polyarteritis, but this phenomenon has not so far been described in children. We report three children with crescentic glomerulonephritis in whom ANCA concentrations were raised at presentation. Two cases were idiopathic, and the other later developed features of Wegener's granulomatosis. In all three, plasma exchange and immunosuppression removed ANCA, but in only one case was there clinical improvement. This child later developed classical nasal lesions of Wegener's granulomatosis associated with a decline in renal function and a rise in ANCA. Plasma exchange and immunosuppression again produced a good clinical response and removed ANCA. This suggests that ANCA is either a marker of disease activity, or is involved in the pathogenesis of disease.

Crescentic glomerulonephritis is characterised by intraglomerular extracapillary cellular proliferation and is a relatively rare pathological finding in childhood. It is often associated with the clinical picture of rapidly progressive renal failure. It may be idiopathic or associated with other diseases such as post-streptococcal glomerulonephritis, infective endocarditis, IgA nephropathy, Goodpasture's syndrome, membranoproliferative glomerulonephritis, systemic lupus erythematosus, Henoch-Schönlein purpura, and other systemic vasculitides. The pathogenesis of the histological lesion is incompletely understood. ${ }^{\prime}$

Microscopic polyarteritis and Wegener's granulomatosis are rare causes of crescentic glomerulonephritis in childhood. Circulating antineutrophil cytoplasm antibody (ANCA) has been described in these disorders in adults, ${ }^{2-6}$ but not so far in children. We describe three children with crescentic glomerulonephritis in whom high concentrations of ANCA were detected both by indirect immunofluorescence by overlaying sera on normal neutrophils and by a solid phase radioimmunoassay.

\section{Case reports}

Case 1. An 11 year old girl presented in acute renal failure after a one week history of vomiting, abdominal pain, and haematuria. She was oliguric, normotensive, and her plasma creatinine concentration was $603 \mu \mathrm{mol} / \mathrm{l}$. Despite having no clinical or bacteriological evidence of a recent streptococcal throat infection, she had a raised antistreptolysin $\mathrm{O}$ titre of $1200 \mathrm{U} / \mathrm{ml}$ (upper limit of normal $400 \mathrm{U} / \mathrm{ml}$ ), a raised anti-DNAase B titre $600-800 \mathrm{U} / \mathrm{ml}$ (upper limit of normal $450 \mathrm{U} / \mathrm{ml}$ ), and a low $\mathrm{C} 3$ concentration of $60 \%$ of reference normal serum (normal range $86-118 \%$ ), suggesting a diagnosis of poststreptococcal glomerulonephritis. She responded to diuretic treatment, but one week after admission her plasma creatinine concentration was still raised at $530 \mu \mathrm{mol} / \mathrm{l}$, and a renal biopsy was performed. The biopsy specimen contained nine glomeruli, all of which had large cellular crescents and hypercellular tufts (fig 1). Immunofluorescence showed the presence of fibrin in the crescents, but no other immunoreactants were detected. Circulating ANCA was shown in her serum by indirect immunofluorescence on normal neutrophils (fig 2), and confirmed by solid phase radioimmunoassay at a titre of $54 \%$ of a positive control (upper limit of normal range $15 \%$ ).

In view of the severity of the histological changes she was treated with prednisolone $2 \mathrm{mg} / \mathrm{kg} / \mathrm{day}$, cyclophosphamide $3 \mathrm{mg} / \mathrm{kg} / \mathrm{day}$, and a course of daily $4000 \mathrm{ml}$ plasma exchanges for five days using 


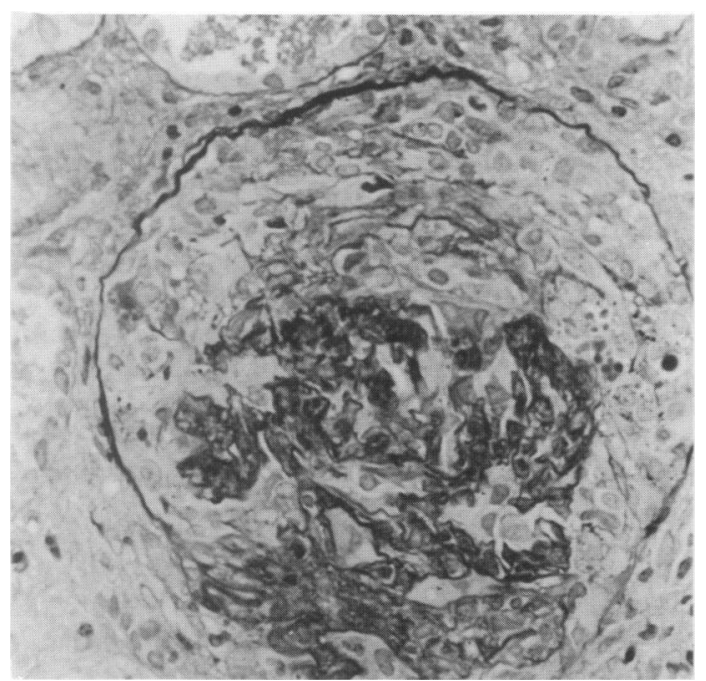

Fig 1 High power section from the renal biopsy specimen of case I showing a glomerulus with a large cellular crescent.

purified protein fraction as replacement with fresh frozen plasma for the last $300 \mathrm{ml}$ of each exchange. After this her urine output increased and her plasma creatinine concentration fell to $120 \mu \mathrm{mol} / \mathrm{l}$. One week later she was discharged home on a reducing dose of immunosuppressives. At this time her ANCA was $11 \%$ of positive control (within the normal range), and her glomerular filtration rate was $118 \mathrm{ml} / \mathrm{min} / 1.73 \mathrm{~m}^{2}$ surface area.

Four months later her glomerular filtration rate had fallen to $70 \mathrm{ml} / \mathrm{min} / 1.73 \mathrm{~m}^{2}$ surface area, and she developed nasal lesions that were histologically compatible with Wegener's granulomatosis. ANCA had risen to $60 \%$ of positive control. Another course of five plasma exchanges combined with cyclophosphamide and steroids again reduced the ANCA concentration to $9 \%$ of positive control and her glomerular filtration rate increased to $99 \mathrm{ml} / \mathrm{min} /$ $1.73 \mathrm{~m}^{2}$ surface area. One year later she remains well on prednisolone $30 \mathrm{mg}$ on alternate days and azathioprine $100 \mathrm{mg} /$ day with no evidence of recurrence of respiratory tract disease. She has a glomerular filtration rate of $94 \mathrm{ml} / \mathrm{min} / 1.73 \mathrm{~m}^{2}$ surface area, and has an ANCA concentration measured by solid phase radioimmunoassay of $<5 \%$ of positive control. Her clinical course and circulating ANCA concentrations are summarised in fig 3 .

Case 2. A 12 year old girl presented with a grand mal convulsion after a six week history of vomiting, malaise, and headaches. She was found to be in
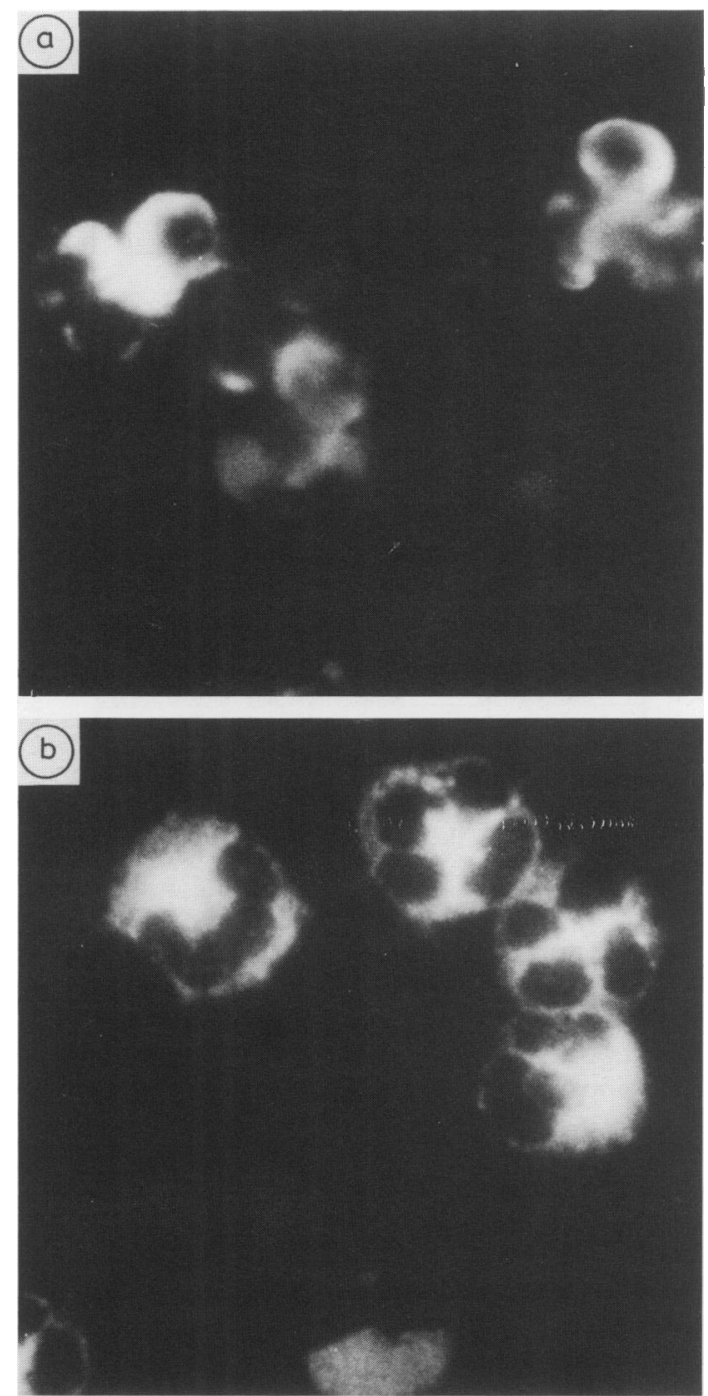

Fig 2 Detection of ANCA by indirect

immunofluorescence. Normal neutrophils are incubated with test serum (diluted 1/8), followed by

fluorescein-isothiocyanate-conjugated rabbit antihuman IgG. (a) Serum from case 1 : (b) serum from an adult case of Wegener's granulomatosis that has been proved on biopsy. Both show fluorescence of neutrophil cytoplasm.

acute renal failure with anuria, fluid overload, hypertension, and her plasma creatinine concentration was $1560 \mu \mathrm{mol} / \mathrm{l}$. She was treated by peritoneal dialysis. Initial investigations, which included a normal renal ultrasound, failed to establish the diagnosis. Serum antistreptolysin $\mathrm{O}$ titre and antiDNAase titre were normal, but C3 was $76 \%$ of 


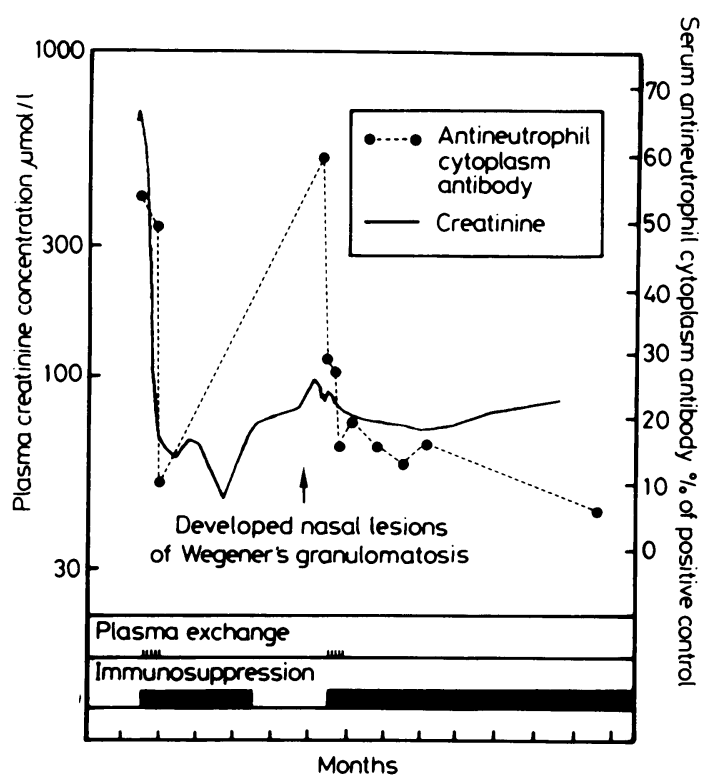

Fig 3 Clinical course of case I showing plasma creatinine concentration and $A N C A$ in relation to treatment.

reference normal serum. She had circulating ANCA shown by indirect immunofluorescence, which when measured by solid phase radioimmunoassay was $41 \%$ of positive control. She remained anuric, and a renal biopsy specimen showed all of 60 glomeruli to be severely damaged with predominantly fibrous crescents and sclerosis and hyalinisation of the tufts. Immunofluorescence showed deposits of IgM. Clq, and $\mathrm{C} 3$ along the capillary loops.

A course of daily plasma exchanges for five days combined with prednisolone $2 \mathrm{mg} / \mathrm{kg} /$ day and cyclophosphamide $3 \mathrm{mg} / \mathrm{kg} /$ day reduced the circulating ANCA to $12 \%$ of positive control, but she remained anuric. She was entered into a programme for continuous ambulatory peritoneal dialysis but developed peritonitis due to Aspergillus and subsequently an acute meningoencephalopathy of unknown aetiology. This failed to respond to broad spectrum antimicrobial treatment, and despite intensive supportive treatment she died of rapidly progressive neurological disease. A postmortem examination was not performed.

Case 3. An 11 year old girl presented with acute renal failure six weeks after a 10 day illness consisting of sore throat, cough, fever, and haematuria. She was oliguric, fluid overloaded, and hypertensive, and her plasma creatinine concentration was $521 \mu \mathrm{mol} / \mathrm{l}$. She was treated by peritoneal dialysis. Initial investigations showed an antistreptolysin $\mathrm{O}$ titre of $400-600 \mathrm{U} / \mathrm{ml}$, an anti-DNAase B titre of $100-200 \mathrm{U} / \mathrm{ml}$, and a normal C3 concentration of $112 \%$ of reference normal serum. She had circulating ANCA detectable by immunofluorescence, which measured by solid phase radioimmunoassay was $32 \%$ of positive control. A biopsy specimen showed $36(95 \%)$ of 38 glomeruli to have predominantly fibrous crescents and advanced global sclerosis. Immunofluorescence showed scanty IgM and C3 deposition along capillary loops. Despite a course of daily plasma exchanges for five days combined with prednisolone $2 \mathrm{mg} / \mathrm{kg} /$ day and cyclophosphamide $3 \mathrm{mg} / \mathrm{kg} / \mathrm{day}$, which removed detectable ANCA, she showed only a transient improvement in renal function. She was entered into a renal replacement programme, ANCA subsequently remained undetectable, and she received a successful renal transplant 13 months after her initial presentation.

\section{Methods}

ANCA was detected by two methods:

(1) INDIRECT IMMUNOFLUORESCENCE ON NORMAI. NEUTROPHIL.S

This technique has been described previously. Normal neutrophils from healthy donors are isolated by density gradient centrifugation and alcoholfixed on glass microscope slides. The slides are incubated for 1 hour at $4^{\circ} \mathrm{C}$ first with dilutions of test or control sera, and then with fluoresceinisothiocyanate-conjugated rabbit antihuman IgG. The slides are then examined under ultraviolet light, and samples are considered positive if most neutrophils show bright fluorescence in the cytoplasm (fig 2), and negative if no staining is present.

\section{(2) SOLID PHASE RADIOIMMUNOASSAY}

This technique has also been described previously. ${ }^{5}$ Neutrophil antigen is prepared from normal donor neutrophils by sonication and centrifugation in sodium acetate $(0.2 \mathrm{~mol} / \mathrm{l}, \mathrm{pH} 4 \cdot 2)$, and the supernatant saved. Wells of plastic microtitre plates are coated with the antigen by incubating with the supernatant for 1 hour at $37^{\circ} \mathrm{C}$. Dilutions of test or control sera are then incubated in the wells for 30 minutes at $37^{\circ} \mathrm{C}$. IgG bound to the wells is detected by a treble-layer technique (mouse monoclonal antihuman $\mathrm{IgG}$, then rabbit antimouse $\mathrm{IgG}$, then ${ }^{125}$ I-labelled goat antirabbit $\operatorname{IgG}$ ), and measured by counting in a gamma counter. The results of this binding are expressed as a percentage of the binding seen for a standard positive serum from an adult case of Wegener's granulomatosis that has been proved on biopsy. 


\section{Discussion}

Crescentic glomerulonephritis is a relatively rare condition in childhood. The severe form can be defined as the presence of crescents in at least $80 \%$ of glomeruli and presents as rapidly progressive glomerulonephritis. Only 10 new cases of this severe form have been admitted to the renal unit of the Hospital for Sick Children, London since June 1985 when we started screening these patients for raised ANCA; during this same period we dialysed 171 children with other causes of acute renal failure. In addition to the three cases reported here, three cases were associated with Henoch-Schönlein syndrome, one with anti-glomerular basement membrane nephritis, one with post-streptococcal glomerulonephritis, and two cases were idiopathic. Only the three cases reported here, however, had raised ANCA at presentation. Although ANCA has been shown in the sera of adult patients with Wegener's granulomatosis and microscopic polyarteritis ${ }^{2-6}$ it has not previously been reported in children. In case 1 , the diagnosis initially appeared to be poststreptococcal glomerulonephritis despite there being no clinical or microbiological evidence of a throat or skin infection. Four months later, however, she developed clinical symptoms which, supported by histological evidence, established the diagnosis as Wegener's granulomatosis. Cases 2 and 3 both appeared to be idiopathic at presentation, but in view of the raised ANCA and the experience from adult cases, might perhaps represent occult microscopic polyarteritis.

The significance of the presence of this antibody is unclear, but a role in the pathogenesis of vasculitis is suggested as its concentration has been shown in adults to decrease as clinical signs of disease activity improve. ${ }^{6}$ In the three children described here, plasma exchange and immunosuppression resulted in an appreciable reduction in concentrations of circulating ANCA, but only in case 1 did this coincide with important clinical improvement. She had the shortest history, and in contrast with the others her renal biopsy specimen showed predominantly cellular crescents. When she later relapsed with deterioration of renal function and the development of the nasal lesions of Wegener's granulomatosis, her circulating ANCA concentration had again risen (to $60 \%$ of positive control), suggesting that the antibody had either a pathogenic role or was a marker of disease activity.

Circulating ANCA should be sought in children with crescentic glomerulonephritis, and when present may be of diagnostic value, pointing to Wegener's granulomatosis or microscopic polyarteritis. It may also be of value in monitoring the effectiveness of treatment in cases with a short duration of symptoms.

We thank S Jones and PD Marshall for help with the ANCA assay. CM Lockwood was supported by a grant from the Wellcome Trust.

\section{References}

1 Southwest Pediatric Study Group. A clinico-pathologic study of crescentic glomerulonephritis in 50 children. Kidney Int 1985;27: 450-8.

2 Davies DJ, Moran JE, Niall JF, Ryan GB. Segmental necrotising glomerulonephritis with anti-neutrophil antibody: possible arbovirus aetiology. $\mathrm{Br}$ Med $J$ 1982;285:606.

3 van der Woude FJ, Rasmussen N, Lobatto S, et al. Autoantibodies against neutrophils and monocytes: tool for diagnosis and marker of disease activity in Wegener's granulomatosis. Lancet 1985;i:425-9.

4 Gross WL, Ludeman G, Kiefer G, Lehmann H. Anticytoplasmic antibodies in Wegener's granulomatosis. Lancet 1986;i:806. Lockwood CM, Bakes D, Jones S, Whitaker KB, Moss DW, Savage COS. Association of alkaline phosphatase with an autoantigen recognised by circulating anti-neutrophil antibodies in systemic vasculitis. Lancet 1987; i: 716-20.

- Savage COS, Winearls CG, Jones S, Marshall PD, Lockwood CM. Prospective study of radioimmunoassay for antibodies against neutrophil cytoplasm in diagnosis of systemic vasculitis. Lancet 1987;i:1389-93.

Correspondence to Dr MDS Walters, Department of Paediatric Nephrology, Institute of Child Health, 30 Guilford Street, London WC1N 1EH.

Accepted 2 February 1988 